\title{
Microsurgical Reconstruction of the Mandible in a Patient with Evans Syndrome: A Case Report and Review of the Literature
}

\author{
Alberto Bedogni, MD ${ }^{1}$ Alexandre Anesi, MD ${ }^{2}$ Andrea Fior, MD ${ }^{1}$ Giordana Bettini, MD ${ }^{3}$ \\ Pier Francesco Nocini, MD, DMD ${ }^{1}$
}

${ }^{1}$ Unit of Oral and Maxillofacial Surgery, Department of Surgery, Azienda Ospedaliera Universitaria Integrata Verona, Verona, Italy

2 Section of Cranio-Maxillo-Facial Surgery, Department of Head \& Neck Surgery, University Hospital of Modena, Modena, Italy

${ }^{3}$ Unit of Maxillofacial Surgery, Department of Neurosciences, University of Padova, Padua, Italy

J Reconstr Microsurg 2013;29:545-550.

\begin{abstract}
Address for correspondence Alberto Bedogni, MD, Unit of Oral and Maxillofacial Surgery, Department of Surgery, Azienda Ospedaliera Universitaria Integrata, Piazzale L.A. Scuro, 10, 37134 - Verona, Italy (e-mail: alberto.bedogni@univr.it).
\end{abstract}

\author{
Abstract \\ Keywords \\ - Evans Syndrome \\ - idiopathic \\ thrombocytopenic \\ purpura \\ - microsurgery \\ - thrombosis \\ - fibula flap
}

In this report, we describe the first successful case of microvascular free tissue transfer in a patient with Evans Syndrome (ES), a rare form of idiopathic thrombocytopenic purpura (ITP) and associated autoimmune hemolytic anemia (AIHA). Microvascular surgery in the setting of ES is likely to have higher complication rates because of the increased risk of postoperative bleeding and free flap thrombosis. The case presented here opens up to the feasibility of microvascular reconstruction of patients with coagulation disorders like ES. Every effort should be made to control for hemolytic, thrombocytopenic, and thrombophilic states associated with ES. In the absence of evidence-based treatment guidelines for ES, personalized treatment protocols with high-dose corticosteroids, immunoglobulin, and postoperative anticoagulation regimen are highly recommended.
Evans syndrome (ES) is a rare autoimmune disorder defined by the combination of idiopathic thrombocytopenic purpura (ITP) and autoimmune hemolytic anemia (AIHA), occasionally with immune neutropenia, in the absence of known underlying etiology. ${ }^{1,2}$ ITP and AIHA may occur simultaneously or sequentially. The peak age of manifestation ranges between 5.5 and 7.7 years and both the sexes are equally affected. ${ }^{1}$ ES has a chronic relapsing course with occasional remission; its clinical features reflect those of hemolytic anemia (pallor, lethargy, and jaundice) and thrombocytopenia (epistaxis, cutaneous or gingival bleeding, hematuria, menorrhagia, and fatigue). First-line therapy of ES is usually done with corticosteroids alone or in combination with intravenous immunoglobulin (IVIG), although the management of ES remains a challenge. ${ }^{1,2}$ Patients with ITP have an increased risk of perioperative bleeding. ${ }^{3,4}$ Since the first report of a successful open cardiac surgery in ITP patients ${ }^{3,5}$ in the 1980 s, no microsurgical free flap procedures have been described in ESpatients so far.

In this report, we describe and discuss the first successful case of microvascular reconstruction of the mandible with free bone flaps in a patient with ES.

\section{Case Report}

A 12-year-old white woman was diagnosed ES in 2003. Initially, a cyclic corticosteroid therapy consisting of 10-day course of prednisone (25 mg, twice daily) was adopted depending on the platelets (PLT) count. The therapy failed to control recurrent episodes of gingival bleeding that occurred during the following years. received

January 5, 2013

accepted after revision

May 17, 2013

published online

June 26, 2013
Copyright (c) 2013 by Thieme Medical Publishers, Inc., 333 Seventh Avenue, New York, NY 10001, USA. Tel: +1(212) 584-4662.
DOI http://dx.doi.org/ 10.1055/s-0033-1348898. ISSN 0743-684X. 
In January 2007, at the age of 16 years, she was presented at our department with a fast-growing painless mass of the left mandibular body ( $\mathbf{- F i g . ~ 1 ) . ~ T h i s ~ l e s i o n ~ c a u s e d ~ t e e t h ~}$ crowding, malocclusion, and facial deformity. Oral and dental care had been neglected for a long time owing to the risk of further hemorrhages. Computed tomographic scans showed a 14-cm-wide multiloculated bone lesion extending from the left mandibular angle to the contralateral premolar region, with cortical interruption ( $\mathbf{- F i g . ~ 1 ) . ~ B l o o d ~ t e s t s ~ r e v e a l e d ~ a ~}$ severe thrombocytopenia (PLT $26 \times 10^{9} / \mathrm{L}$ ) and a prolonged activated partial thromboplastin time (1.7 ratio) that was linked to the presence of antiphospholipid antibodies (aPL). AIHA disorder proved mild. A cushingoid state was present as a result of the chronic steroid therapy that was tapered and then interrupted. The patient was started on a 4-day course of dexamethasone (1-day upload course of $40 \mathrm{mg} /$ four times daily, then $40 \mathrm{mg}$ /four times daily), with a rapid increase of the PLT count $\left(243 \times 10^{9} / \mathrm{L}\right)$, which remained stable above $100 \times 10^{9} / \mathrm{L}$ for several weeks. Within a normalized risk of bleeding, the patient underwent a biopsy of the bone lesion. On the basis of the pathology report, a conclusive diagnosis of aggressive central giant cell granuloma (CGCG) was made, once hyperparathyroidism was ruled out. Furthermore, den-
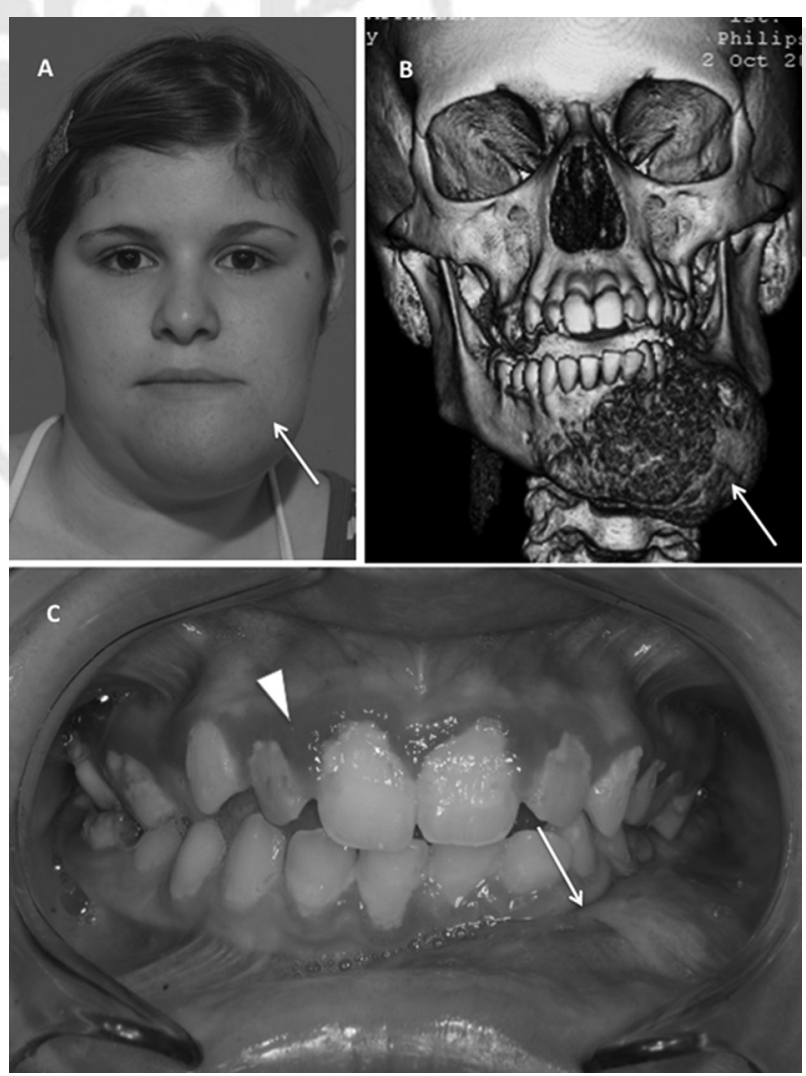

Fig. 1 Preoperative. (A) Frontal view of the patient: gross deformity of the mandible and facial asymmetry due to abnormal tumor-related skeletal growth (white arrow). (B) Computed tomography (threedimensional computed tomography): extensive bone lesion involving the entire horizontal branch of the mandible; note the multiple fenestrations of the outer cortical plate (white arrow). (C) Intraoral view: obliteration of the left mucosal vestibule, teeth crowding (white arrow), and severe periodontal disease (white arrowhead). tal treatments were safely performed on an outpatient basis before the patient underwent subtotal mandibulectomy in June 2007. The 14-cm-long mandibular defect was immediately reconstructed with an osteomuscular fibular bone flap harvested from the right leg. The peroneal artery and accompanying veins were anastomosed to the right facial artery and vein and to the external jugular vein (-Fig. 2). No lifethreatening intraoperative bleeding occurred. At the end of the procedure, the PLT count was $138 \times 10^{9} / \mathrm{L}$ and hemoglobin was $8.90 \mathrm{~g} / \mathrm{L}$. Postoperative AIHA and ITP were controlled with the use of methylprednisolone intravenous dextran $40,000 \mathrm{pm}$ at a dose of $20 \mathrm{~mL} / \mathrm{h}$ and low-molecular-weight heparin (2,850 IU subcutaneous) were administered daily for 5 days. The patient's postoperative course was uneventful and the patient was discharged 18 days after surgery with a PLT count of $137 \times 10^{9} / \mathrm{L}$.

The patient remained stable without major bleeding up to the age of 18 when she was asked for a full-arch dental rehabilitation (-Fig. 3). After consultation with the Hematology Department, a second vascularized fibula flap was planned to reconstruct the alveolar bone region and fill-up the vertical gap of the mandible. In November 2009, the patient underwent free fibula flap transfer from the left leg.

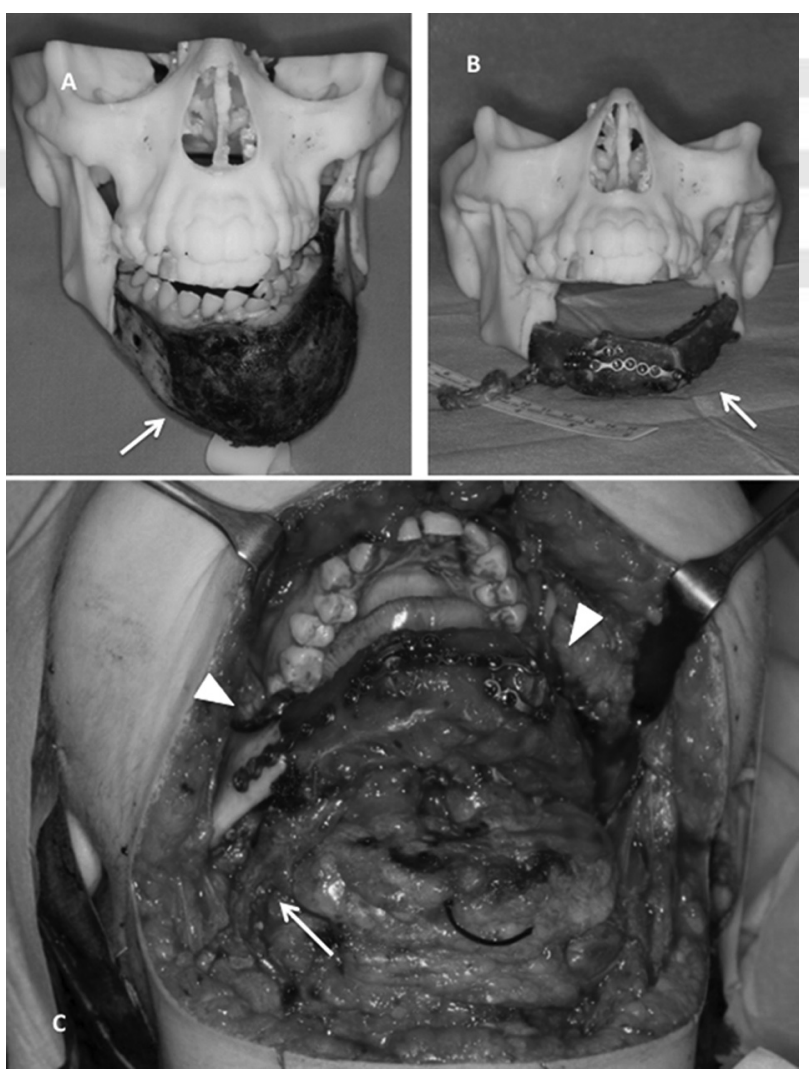

Fig. 2 First operation. (A) Specimen: intraoperative confirmation of the margins of mandible resection onto the stereolithographic model (white arrow). (B) Fibula flap: verification of the three-dimensional skeletal relationships of the new mandible before flap inset (white arrow). (C) View of the reconstructed mandible after flap inset and completion of microvascular anastomoses (white arrow). Two 2.0-mm locking plates (2.0-mm Mandible Locking Plates System, Synthes, Inc., West Chester, PA) were used to fix each bone segment and the mandibular stumps (white arrowhead). 

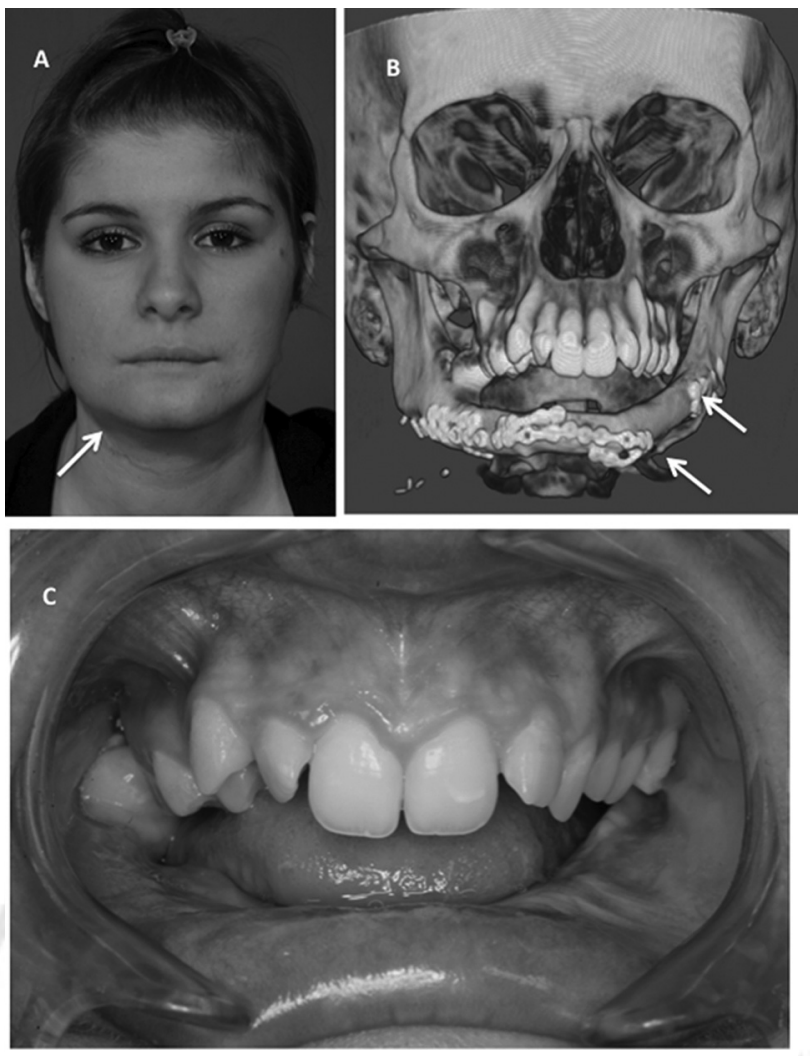

Fig. 3 18-month follow-up (first operation). (A) Frontal view of the patient: re-established symmetry of the oval; note the shortened lower third of the face because of insufficient mandibular bone height (white arrow). (B) Three-dimensional computed tomography: fully restored mandibular bone buttressing and complete bone union between the fibular segments and the residual mandibular stumps (white arrow). (C) Intraoral view: stable mucosal healing of the reconstructed mandible.

Surgery was performed by the same operators (A.B., A.F., S.V., and G.B.), and the previously adopted operative and medical protocols were used. Postoperative course was uneventful and the patient was discharged 14 days after surgery with a PLT count of $146 \times 10^{9} / \mathrm{L}$. Two more surgeries were required for the completion of the dental rehabilitation protocol. The patient was free of disease at 3-year follow-up without any signs of AIHA and ITP (-Fig. 4).

\section{Discussion}

ES is a rare form (1\%) of ITP and associated AIHA. ${ }^{2}$ The increased risk of bleeding and life-threatening hemorrhages that is secondary to the accelerated PLT destruction caused by antiplatelet antibodies ${ }^{1,6}$ usually contraindicate major surgery in ES patients.

In the present case both diagnosis and treatment of the mandibular lesion have been postponed for more than 3 years since the initiation of the disease process, due to the fear of bleeding.

CGCG of the jaws is a reparative nonneoplastic, nonhyperparathyroidism-related process, whose treatment is usually
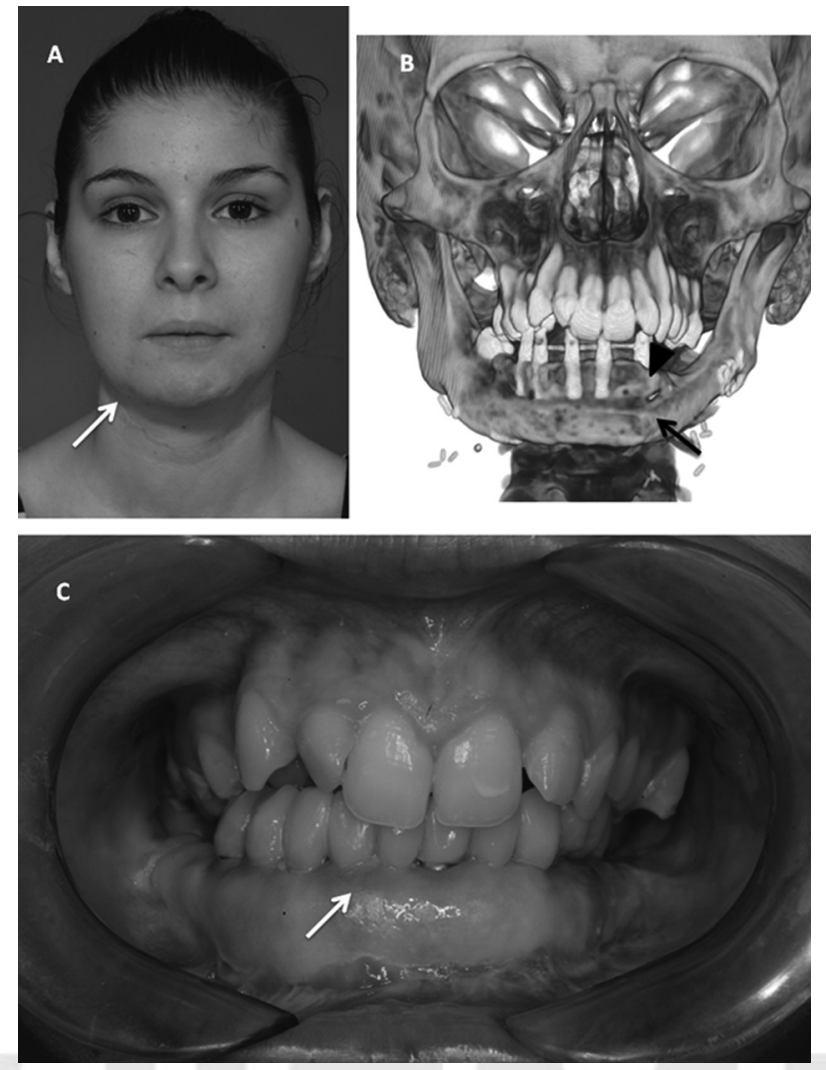

Fig. 4 3-year follow-up (second operation). (A) Frontal view of the patient: improved symmetry of the lower third of the face following augmentation of the mandible with the second fibula flap and implantsupported prosthetic restoration (white arrow). (B) Three-dimensional computed tomography: the initial fibula flap (basal bone) (black arrow) and the second one (alveolar bone) (black arrowhead) fused together to provide a nearly natural shape of the reconstructed mandible. (C) Intraoral view: healthy peri-implant (lower jaw) and periodontal seal (upper jaw); of note, the peri-implant seal was obtained by means of secondary split-thickness skin grafting at the time of second-stage implant surgery (white arrow).

conservative (i.e., curettage, corticosteroid injections, calcitonin, and interferon alfa). ${ }^{7}$ Aggressive forms often require a radical approach, as in this case where facial disfigurement and malocclusion were present. ${ }^{8}$ Fibula is the workhorse flap in pediatric mandibular reconstruction when long defects have to be spanned. ${ }^{9}$

Bleeding disorders and thrombophilia are considered among the most relevant factors that can influence the outcome of microsurgical reconstruction. ${ }^{10-12}$

A narrative review of the available literature turned out few case reports describing free flap failure in patients with undiagnosed hypercoagulation disorders, who underwent major head and neck reconstruction. ${ }^{13-17}$ These authors state that hypercoagulation disorders may represent an independent factor affecting free flap success. ${ }^{18}$

A recent internal survey from Wang et $\mathrm{al}^{19}$ found an increased risk of free flap failure also in patients with known thrombophilic conditions; nonetheless, microsurgical reconstruction was not contraindicated in those patients when an 
accurate preoperative work-up and perioperative treatment of thrombophilia is performed, according to the American guidelines of College of Chest Physicians. ${ }^{20}$

Thrombophilia increases the risk of thrombosis and free flap failure also in ES patients, especially when prolonged activated partial thromboplastin time is present, which is often related to aPLs, as in this case. ${ }^{2,6}$

A variety of preoperative treatment strategies have been proposed in ES to boost PLT count before intervention and to minimize perioperative hemorrhages, including preoperative or concomitant splenectomy, corticosteroids medication, preoperative and/or postoperative IVIG, PLT transfusion, and immunosuppressive agent (danazol). ${ }^{21,22}$ First-line therapy for nonemergent indications is based on corticosteroids and/or IVIG. In emergent setting, blood and PLT transfusion may be administered to alleviate symptoms. Initial therapy for nonemergent indications is based on orally prednisone (1 $\mathrm{mg} / \mathrm{kg}$ daily) for 3 or 4 weeks or prednisolone $(1-4 \mathrm{mg} / \mathrm{kg}$ daily).

Only 10 to $30 \%$ of ES patients on long-term corticosteroid therapy achieve stable remission and thrombocytopenia usually recurs in most cases after discontinuation. ${ }^{1,2}$ When steroids are ineffective or toxic, the most common first-line therapy is concomitantly used of IVIG $(0.4 \mathrm{~g} / \mathrm{kg} / \mathrm{daily}$ for 4 days). ${ }^{1}$ Second-line therapy includes immunosuppressive agents (cyclosporine, mycophenolate mofetil, and danazol), the monoclonal antibody rituximab and chemotherapy (vincristine). Splenectomy is recommended in persistent ITP patient in case of a remission period less than 1 year after diagnosis, when the PLT count is consistently less than $20,000 \times 10^{9} / \mathrm{L}$ or when the patient is not responding to therapy $^{21}$; long-term remission in ES after splenectomy is less frequent than in uncomplicated ITP. Stem cell transplantation offers a further chance for severe and refractory cases. ${ }^{1}$

Prolonged activated partial thromboplastin time in ITP patients is often related to aPL presence and give rise to a thrombotic risk (arterial and venous thrombosis, recurrent fetal loss). ${ }^{2,6}$

As no successful experience is reported in ES patients who are candidates for major head and neck surgical resections and microsurgical reconstructions, we followed the recommendations of the British Haematology task force $^{23}$ and maintained PLT count above $100,000 \times 10^{9} / \mathrm{L}$ using perioperative high-dose corticosteroids. ${ }^{2}$ of importance, PLT counts should not exceed $200,000 \times 10^{9} / \mathrm{L}$ to minimize the associated thrombophilic risk. High-dose dexamethasone was effective to boost PLT count before intervention. Immunomodulating agents and IVIG were also prepared to be used in case of necessity, to diminish the substantial risk of thrombosis associated with aPLs in the postoperative. Nevertheless, thromboprophylaxis with daily subcutaneous injection of low-molecular-weight heparin was enough to prevent from vessel thrombosis and flap failure in the present case.

In conclusion, microvascular free flap surgery is feasible also in ES patients with autoimmune clotting disorders. ES patients who are candidates to microsurgical reconstructions should be carefully assessed for their hemolytic, thrombocy- topenic, and thrombophilic states and treated accordingly to minimize the risk of free flap failure.

\section{Competing Interests \\ None declared.}

\section{Funding}

None.

\section{References}

1 Norton A, Roberts I. Management of Evans syndrome. Br J Haematol 2006;132(2):125-137

2 Cines DB, Bussel JB. How I treat idiopathic thrombocytopenic purpura (ITP). Blood 2005;106(7):2244-2251

3 Marõnas JM, Llamas P, Caffarena JM. Mitral valve replacement and splenectomy in a patient with chronic idiopathic thrombocytopenic purpura. Thorac Cardiovasc Surg 1982;30(6):407-408

4 Nakajima T, Iyoda A, Iizasa T, Saitoh Y, Hiroshima K, Fujisawa T. Surgical treatment of a superior sulcus tumor in a patient with idiopathic thrombocytopenic purpura: report of a case. Surg Today 2005;35(12):1078-1080

5 Mori Y, Hadama T, Takasaki H, et al. Aortic valve replacement and splenectomy in a patient with chronic idiopathic thrombocytopenic purpura-preoperative management with high-dose gammaglobulin. Heart Vessels 1991;6(2):121-124

6 Diz-Küçükkaya R, Hacihanefioğlu A, Yenerel M, et al. Antiphospholipid antibodies and antiphospholipid syndrome in patients presenting with immune thrombocytopenic purpura: a prospective cohort study. Blood 2001;98(6):1760-1764

7 de Lange J, van den Akker HP, van den Berg H. Central giant cell granuloma of the jaw: a review of the literature with emphasis on therapy options. Oral Surg Oral Med Oral Pathol Oral Radiol Endod 2007;104(5):603-615

8 Bataineh AB, Al-Khateeb T, Rawashdeh MA. The surgical treatment of central giant cell granuloma of the mandible. J Oral Maxillofac Surg 2002;60(7):756-761

9 Cordeiro PG, Disa JJ, Hidalgo DA, Hu QY. Reconstruction of the mandible with osseous free flaps: a 10-year experience with 150 consecutive patients. Plast Reconstr Surg 1999;104(5):1314-1320

10 Chen HC, Coskunfirat OK, Ozkan O, et al. Guidelines for the optimization of microsurgery in atherosclerotic patients. Microsurgery 2006;26(5):356-362

11 Baumeister S, Follmar KE, Zenn MR, Erdmann D, Levin LS. Strategy for reoperative free flaps after failure of a first flap. Plast Reconstr Surg 2008;122(3):962-971

12 Handschel J, Burghardt S, Naujoks C, Kübler NR, Giers G. Parameters predicting complications in flap surgery. Oral Surg Oral Med Oral Pathol Oral Radiol 2013;115(5):589-594

13 Shaw RJ, Rogers SN. Venous microvascular anastomotic failure due to prothrombin gene mutation. Plast Reconstr Surg 2005;115(5): 1451-1452

14 Salgarello M, Cervelli D, Barone-Adesi L. A massive arterial thrombosis of a free anterolateral thigh flap in a patient with antiphospholipid syndrome. Microsurgery 2008;28(6):447-451

15 Davison SP, Kessler CM, Al-Attar A. Microvascular free flap failure caused by unrecognized hypercoagulability. Plast Reconstr Surg 2009;124(2):490-495

16 Asai E, Okouchi M, Momiyama M, Kajikawa A, Ueda K. Free flap failure in an anticardiolipin antibody-positive patient with neoplasm-a case report. Microsurgery 2010;30(3):238-241

17 Loeffelbein DJ, Baumann CM, Mücke T, Wolff KD, Hölzle F, Kesting MR. Sticky platelet syndrome as a possible cause for free flap failure-a case report. Microsurgery 2010;30(6):466-468 
18 Herrera FA, Lee CK, Kryger G, et al. Microsurgery in the hypercoagulable patient: review of the literature. J Reconstr Microsurg 2012;28(5):305-312

19 Wang TY, Serletti JM, Cuker A, et al. Free tissue transfer in the hypercoagulable patient: a review of 58 flaps. Plast Reconstr Surg 2012;129(2):443-453

20 Bates SM, Greer IA, Pabinger I, Sofaer S, Hirsh J; American College of Chest Physicians. Venous thromboembolism, thrombophilia, antithrombotic therapy, and pregnancy: American College of Chest Physicians Evidence-Based Clinical Practice Guidelines (8th Edition). Chest 2008;133:844S-846S
21 George JN. Idiopathic thrombocytopenic purpura in adults: current issues for pathogenesis, diagnosis and management. Hematol J 2004;5(Suppl 3):S12-S14

22 Schmidt RE, Budde U, Schäfer G, Stroehmann I. High-dose intravenous gammaglobulin for idiopathic thrombocytopenic purpura. Lancet 1981;2(8244):475-476

23 British Committee for Standards in Haematology General Haematology Task Force. Guidelines for the investigation and management of idiopathic thrombocytopenic purpura in adults, children and in pregnancy. Br J Haematol 2003;120(4): 574-596
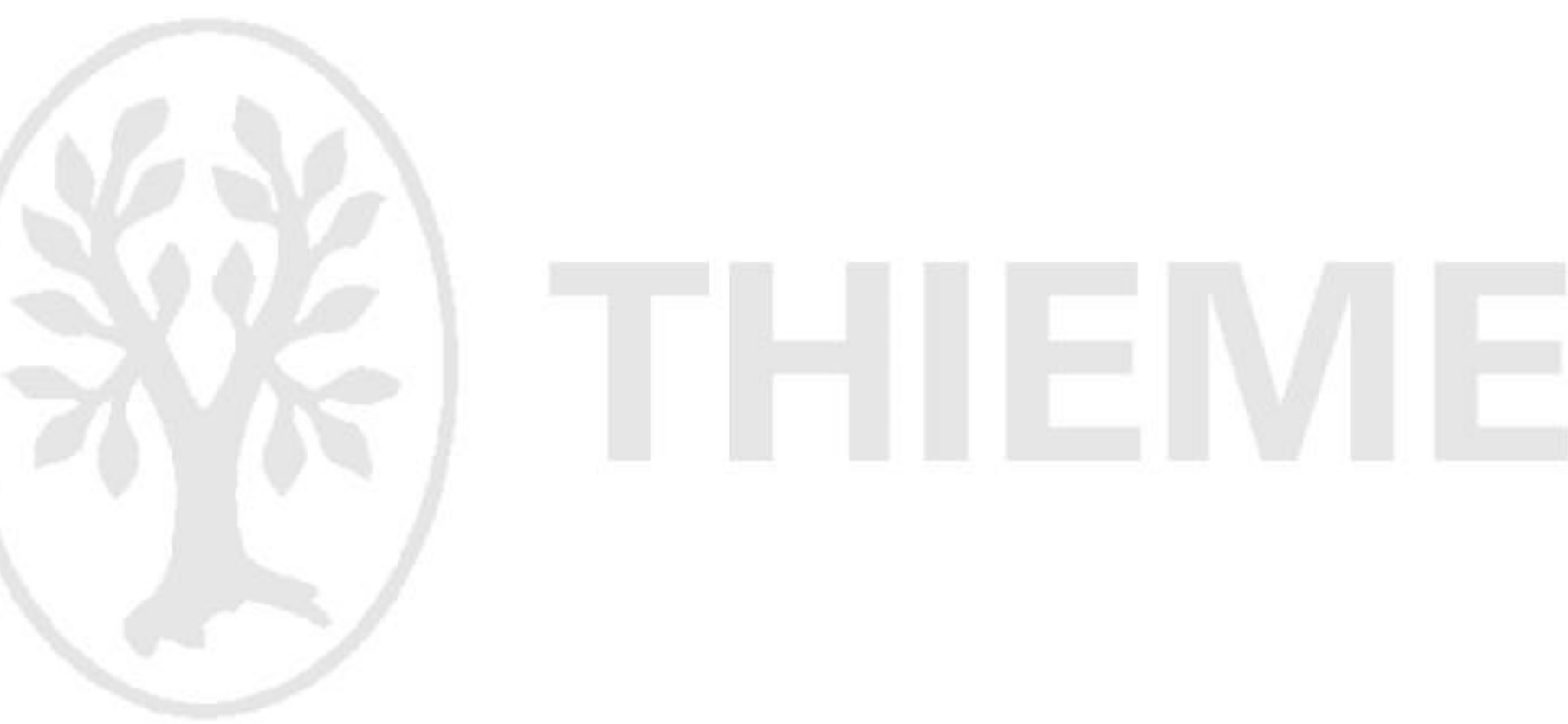

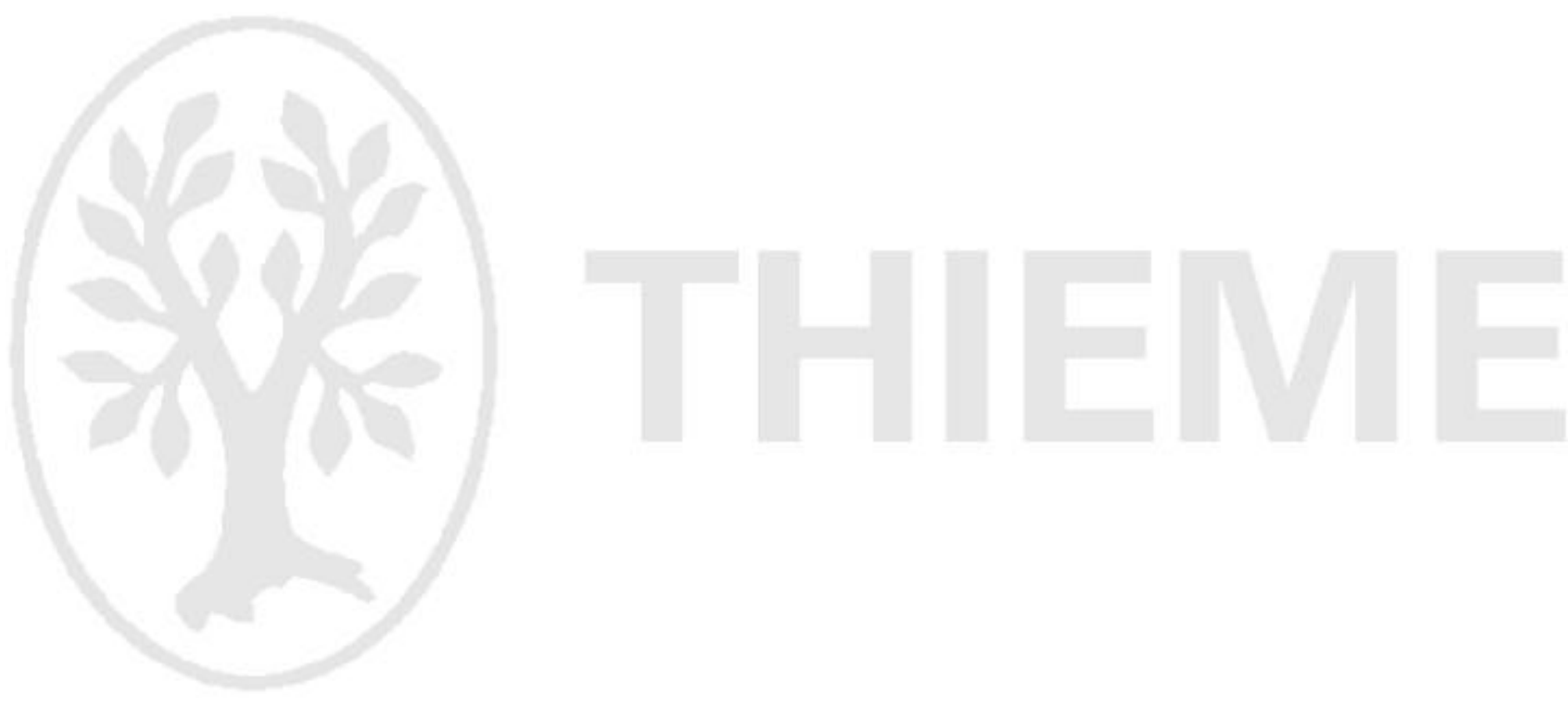